Journal of Computer Science 4 (7): 591-599, 2008

ISSN 1549-3636

(C) 2008 Science Publications

\title{
Skeleton Primitive Extraction Method on Textures with Different Nonlinear Wavelets
}

\author{
${ }^{1}$ V. Vijaya Kumar, ${ }^{2}$ U.S.N. Raju, ${ }^{3}$ P. Premchand and ${ }^{4}$ A. Suresh \\ ${ }^{1,2}$ Department of Computer Science Engineering and Information Technology, \\ GIET, Rajahmundry, A.P., India \\ ${ }^{3}$ Department of Computer Science Engineering, Osmania University, Hyderabad, A.P., India \\ ${ }^{4}$ Indian Railway Service and JNT University Research Scholar, A.P., India
}

\begin{abstract}
Problem Statement: A novel method for dominant skeleton extraction of textures using different nonlinear wavelet transforms is proposed in this study. In the present study $3 \times 3$ masks are used for extraction of skeleton primitives. For a $3 \times 3$ skeleton primitive there will be $2^{9}$ skeleton primitive combinations. But the present study considered a skeleton primitive for the skeletonization purpose if and only if its center pixel is one and skeleton primitives are represented by the corresponding skeleton primitive weight. By this, there will be $2^{8}$ combinations of skeleton primitives. Approach: The skeleton primitive are used for evaluating skeleton points. The skeleton of an object has the property that it is reduced to one point when the skeleton primitive used for the skeletonization is exactly homothetic to the object. The dominant skeleton subset is evaluated by counting the skeleton points. The skeleton subset that leads to the least skeleton points will be the resultant skeleton subset. The present study classified the textures based on two methods. In the first method textures are classified based on skeleton primitive weight, which is nothing but based on skeleton primitive combination. In the second method classification is made based on distance function of skeleton points. Results: The proposed method is applied on 24 Brodatz textures using the three nonlinear wavelet transformed textures. By this the dominant skeleton primitive weight is obtained for each texture. Based on the number of skeleton points distance measures are calculated based on which texture classification is obtained. Conclusions: The two methods are applied on Brodatz textures using different nonlinear wavelet transforms which classified the textures. The first method is appropriate if one need to classify based on skeleton subsets. The second method is appropriate if the classification is to be done based on least number of skeleton points.
\end{abstract}

Keywords: Dominant skeleton subset, combinations of skeleton primitives, homothetic, skeleton points, nonlinear wavelets

\section{INTRODUCTION}

Texture is the term used to characterize the surface of a given phenomenon in an image and it is undoubtedly one of the principal features used in image processing. The different texture analysis methods are structural, statistical, model based and transform based methods. Structural approaches represent texture by well defined primitives (microtexture) and a hierarchy of spatial arrangements (macrotexture) of those primitives. Statistical approaches represent the texture indirectly by the non-deterministic properties that govern the distributions and relationships between the grey levels of an image. Model based texture analysis using fractal and stochastic models, attempt to interpret an image texture by use of generative image model and stochastic model respectively. Transform methods of texture analysis, such as Fourier and wavelet transforms ${ }^{[1-3]}$ represent an image in a space whose coordinate system has an interpretation that is closely related to the characteristics of a texture. Texture classification plays an important role in the interpretation and analysis of terrain images, as well as radiographic and microscopic images. Method of texture classification can be broadly divided into two major categories. The first is texture segmentation, based on features with a high degree of spatial localization e.g. with some form of edge detection. The second approach to texture classification is based on a discrimination function using several texture characteristics. Scientists and practitioners have made great efforts in developing advance classification

Corresponding Author: V. Vijaya Kumar, Department of Computer Science Engineering and Information Technology, GIET, Rajahmundry, A.P., India 
approaches and techniques for improving classification $\operatorname{accuracy}^{[4-6]}$.

Study of patterns on textures is recognized as an important step in characterization and classification of textures. Textures are classified recently by various pattern methods: preprocessed images ${ }^{[13]}$, long linear patterns ${ }^{[8,12]}$ and edge direction movements ${ }^{[7]}$, Avoiding Complex Patterns ${ }^{[11]}$, marble texture description ${ }^{[10]}$ etc. Textures are also described and classified by using various wavelet transforms: One based on primitive patterns $^{[14]}$ and another based on statistical parameters $^{[9]}$.

Skeletonization is a global space domain technique for shape representation ${ }^{[15]}$. It has been studied extensively since skeletons have attractive properties which make them suitable for structural pattern recognition ${ }^{[15,16]}$. There are two types of skeletonization methods: Pixel-based and non-pixel-based. In a pixelbased method, all pixels inside a shape are used in the skeletonization process. Pixel based methods often use thinning techniques ${ }^{[16,17]}$ or distance transforms ${ }^{[17,18]}$. In a non-pixel-based method, only the contour pixels of a shape are used for skeletonization. The skeleton of the shape is analytically derived from its contour ${ }^{[19,20]}$. Skeletonization has been a part of image processing for a wide variety of applications ${ }^{[21]}$. Digital skeletons, generated by thinning algorithms, are often used to represent objects in a binary digital image for shape analysis and classification. Thinning is a process of reducing patterns to their skeleton; however, skeleton is defined as a set of thin lines, arcs and curves (usually one pixel thick), which are connected with each other in such a way that the geometrical and topological properties of its originating object must be preserved.

Wavelet analysis is known as a linear tool. However, it is to be recognized that nonlinear extensions are possible $\mathrm{e}^{[23-25]}$. The lifting scheme, recently introduced by Sweldens ${ }^{[22,26,27]}$ for a predecessor of this scheme, known as a ladder network, has provided a useful tool for constructing nonlinear wavelet transforms. The enormous flexibility and freedom that the lifting scheme offers has challenged researchers to develop various nonlinear wavelet transforms ${ }^{[23-25]}$. Here we present a family of nonlinear wavelets based on morphological operators. The simplest nontrivial example of a morphological wavelet is the so called morphological Haar wavelet. The morphological haar wavelets are of two types: Morphological Haar Erosion Wavelet (MHEW) and Morphological Haar Dilation Wavelet (MHDW). The other nonlinear wavelet is Morphological Binary Wavelet transform (MBW).

\section{MATERIALS AND METHODS}

On a $3 \times 3$ mask by assuming center pixel as one always, one can have $2^{8}$ combinations. However the present study has not considered the following skeleton primitive as shown in Fig. 1 for extracting skeleton primitives. By this there will be a total of 255 skeleton primitives on a $3 \times 3$ mask. These skeleton primitives are used for evaluating skeleton points. The skeleton primitives are represented by weight based system as shown in Fig. 2 which are called as skeleton primitive weight (SPW). Some of the skeleton primitive elements with their weights are represented in the Fig. 3.

\begin{tabular}{|l|l|l|}
\hline 0 & 0 & 0 \\
\hline 0 & 1 & 0 \\
\hline 0 & 0 & 0 \\
\hline
\end{tabular}

Fig. 1: Skeleton primitive that is not been considered for skeletonization

\begin{tabular}{|c|c|c|}
\hline $\mathrm{p} \times 2^{0}$ & $\mathrm{p} \times 2^{1}$ & $\mathrm{p} \times 2^{2}$ \\
\hline $\mathrm{p} \times 2^{4}$ & & $\mathrm{p} \times 23$ \\
\hline $\mathrm{p} \times 2^{5}$ & $\mathrm{p} \times 2^{6}$ & $\mathrm{p} \times 2^{7}$ \\
\hline
\end{tabular}

Fig. 2: Skeleton primitive weight representation

\begin{tabular}{|l|l|l|}
\hline 1 & 1 & 0 \\
\hline 0 & 1 & 0 \\
\hline 0 & 0 & 0 \\
\hline
\end{tabular}

(a)

\begin{tabular}{|l|l|l|}
\hline 1 & 1 & 1 \\
\hline 1 & 1 & 0 \\
\hline 0 & 0 & 0 \\
\hline
\end{tabular}

(d)

\begin{tabular}{|c|c|c|}
\hline 1 & 0 & 1 \\
\hline 0 & 1 & 0 \\
\hline 1 & 0 & 1 \\
\hline
\end{tabular}

(g)

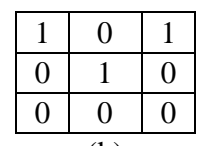

(b)

\begin{tabular}{|l|l|l|}
\hline 1 & 1 & 1 \\
\hline 1 & 1 & 0 \\
\hline 0 & 0 & 0 \\
\hline
\end{tabular}

(e)

\begin{tabular}{|l|l|l|}
\hline 1 & 1 & 1 \\
\hline 0 & 1 & 1 \\
\hline 0 & 1 & 1 \\
\hline
\end{tabular}

(h)

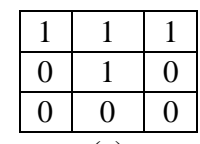

(c)

\begin{tabular}{|l|l|l|}
\hline 1 & 1 & 1 \\
\hline 1 & 1 & 1 \\
\hline 1 & 0 & 0 \\
\hline
\end{tabular}

(f)

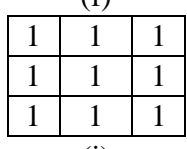

(i)
Fig. 3: Representation of Skeleton primitives with corresponding weights (a) 3 (b) 5 (c) 7 (d) 23 (e) 31 (f) 63 (g) 165 (h) 207 (i ) 255

We propose in this study a novel method of the texture primitive description that requires no assumption on the distribution of grain sizes or the granulometric moments of the primitives. We employ the morphological skeleton for this method. The most commonly employed morphological skeleton of a binary object is explained intuitively as follows: At first 
we locate the largest magnification included within the object and cover the object by sweeping the magnification within the object. Then gradually smaller magnifications are employed for covering the residual area until the whole object is covered. The skeleton varies depending on the shape of the skeleton primitive. If the skeleton primitive is homothetic to the object, the object is covered with only one magnification of the skeleton primitive. In this case the skeleton is reduced to one point. We consider here obtaining the skeleton from a binary texture. It is derived from the above property that the total number of pixels within the skeleton is the minimum when the skeleton primitive is homothetic to the primitive, if we assume that the texture is composed of one primitive, i.e. contains grains that are magnifications of the primitive. This indicates that the primitive is described by the optimal skeleton primitive minimizing the total number of pixels within the skeleton. This primitive description method has an advantage that no assumption on the sizing distribution of grains in the texture is required.

Skeletonization is done according to Algorithm 1. The proposed texture skeleton primitive extraction method on nonlinear wavelets is given in the form of flowchart in Fig. 4. The three nonlinear wavelet transforms used in the present study are MHEW, MHDW and MBW.

\section{Algorithm 1: To find Skeleton of an image Begin \\ 1. Let $k=1$ and $\mathrm{A}$ be the image. \\ 2. $E(A, k B)$,Erode the image $\mathrm{k}$ number of times with skeleton primitive $\mathrm{B}$. \\ 3. $O(A, B)$ Open the image with skeleton primitive B. \\ 4. Find the $k^{\text {th }}$ Skeleton Subset $S_{k}=E(A, k B)$ - $O(E(A, k B)$. \\ 5. $k=k+1$ \\ 6. $\mathrm{T}(\mathrm{A})=\mathrm{E}(\mathrm{A}, \mathrm{kB})$. \\ 7. If $(T(A) \neq \varphi)$ goto 4 . \\ 8. $S K(A, B)$ is the union of all the Skeleton Subsets $S_{k}(A)$. \\ End}

\section{RESULTS}

Evaluation: The proposed Texture Skeleton Primitive extraction method on nonlinear wavelets is applied on 24 Brodatz textures ${ }^{[28]}$ shown in Fig. 5, using 255 skeleton primitive combinations. The process of applying all skeleton primitive combinations for skeletonization is tedious; however it gives accurate result i.e. the exact combination of skeleton subset. The Table 1,2 and 3 indicates the dominant skeleton primitive and the corresponding $S P W$ for all 24 Brodatz textures using MHEW, MHDW and MBW wavelet transform respectively.

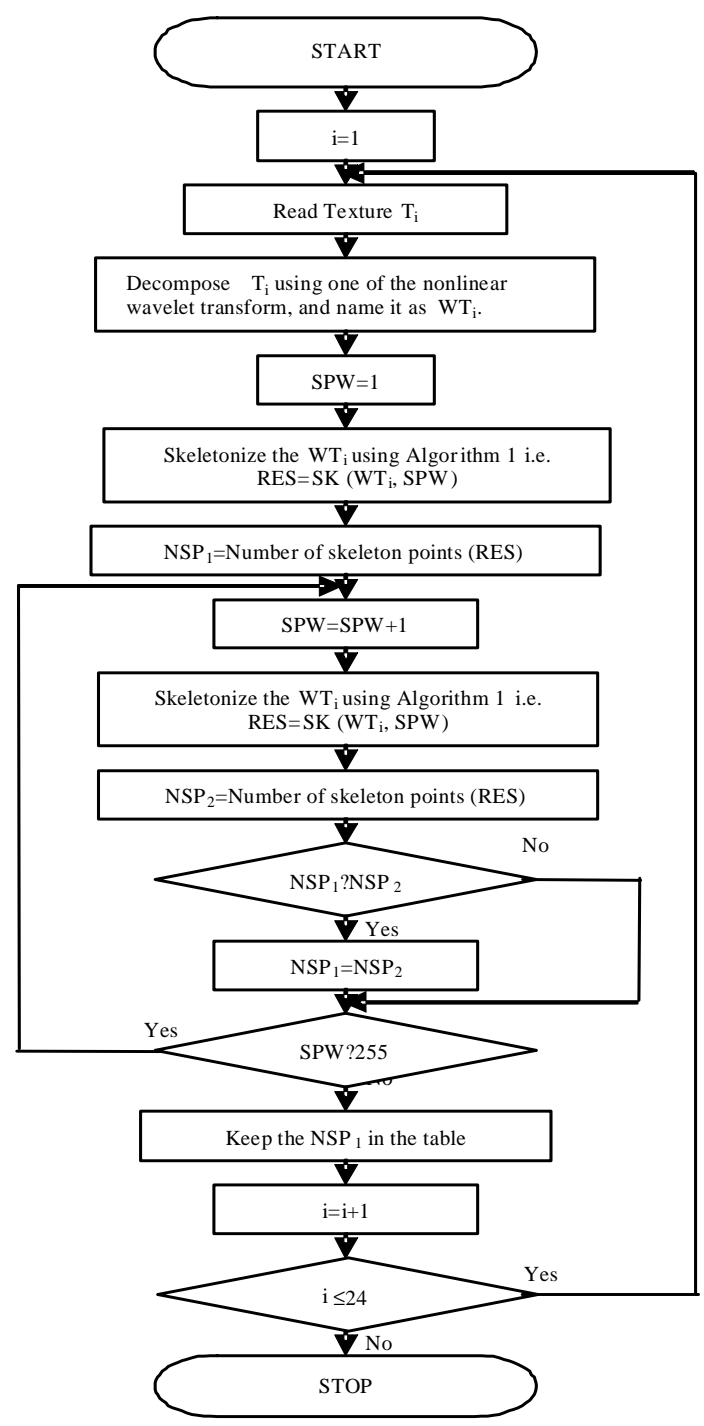

Fig. 4: Flow chart for texture skeleton primitive extraction using nonlinear wavelets.

Based on these results, the textures are classified. From the number of skeleton points, the distance measures are calculated and shown in Table 4, 5 and 6 for the MHE, MHD and MB wavelets respectively. The textures are classified based on these distance measures also. The classification results with lag values 30 and 50 are calculated and are shown in Tables 7 and 8. 


\section{DISCUSSION}

From Table 1, 2 and 3 it is clearly evident that a total of 17, 20 and 18 textures are showing a dominant skeleton primitive with a weight of 255 for MHE, MHD and $\mathrm{MB}$ wavelet transforms respectively as show below.

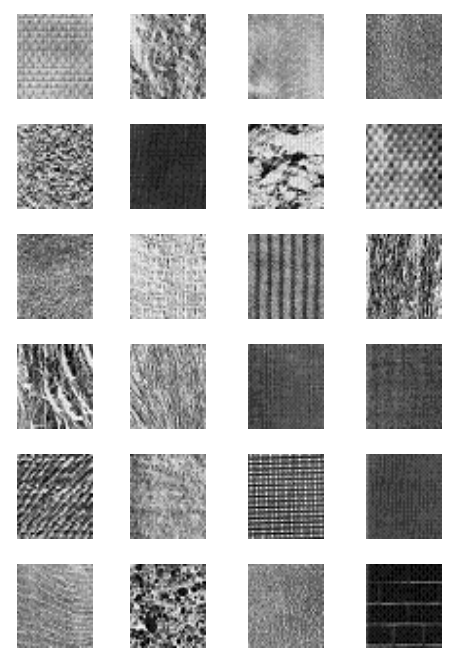

Fig. 5: Twenty four textures from Brodatz album. Row 1: $\mathrm{D}_{1}, \mathrm{D}_{2}, \mathrm{D}_{3}, \mathrm{D}_{4}$. Row 2: $\mathrm{D}_{5}, \mathrm{D}_{6} . \mathrm{D}_{7}, \mathrm{D}_{8}$. Row 3: $\mathrm{D}_{9}, \mathrm{D}_{10}, \mathrm{D}_{11}, \mathrm{D}_{12}$. Row 4: $\mathrm{D}_{13}, \mathrm{D}_{15}, \mathrm{D}_{16}, \mathrm{D}_{17}$. Row 5: $\mathrm{D}_{18}, \mathrm{D}_{19}, \mathrm{D}_{20}, \mathrm{D}_{21}$. Row 6: $\mathrm{D}_{22}, \mathrm{D}_{23}, \mathrm{D}_{24}$, $\mathrm{D}_{25}$.

Table 1: Textures with corresponding skeleton primitive weights by using MHEWT

\begin{tabular}{lll}
\hline Texture & $\begin{array}{l}\text { No. of skeleton } \\
\text { points }\end{array}$ & $\begin{array}{l}\text { Dominant skeleton } \\
\text { primitive weight }\end{array}$ \\
\hline$D_{1}$ & 3940 & 255 \\
$D_{2}$ & 4265 & 255 \\
$D_{3}$ & 6881 & 19 \\
$D_{4}$ & 7155 & 223 \\
$D_{5}$ & 4428 & 255 \\
$D_{6}$ & 3904 & 24 \\
$D_{7}$ & 3717 & 255 \\
$D_{8}$ & 2222 & 255 \\
$D_{9}$ & 6182 & 255 \\
$D_{10}$ & 5600 & 255 \\
$D_{11}$ & 6379 & 255 \\
$D_{12}$ & 4532 & 255 \\
$D_{13}$ & 3850 & 255 \\
$D_{15}$ & 6020 & 255 \\
$D_{16}$ & 7648 & 255 \\
$D_{17}$ & 7550 & 139 \\
$D_{18}$ & 3822 & 255 \\
$D_{19}$ & 5321 & 255 \\
$D_{20}$ & 2688 & 255 \\
$D_{21}$ & 2974 & 24 \\
$D_{22}$ & 5384 & 206 \\
$D_{23}$ & 3429 & 255 \\
$D_{24}$ & 6989 & 66 \\
$D_{25}$ & 3980 & 255 \\
\hline
\end{tabular}

Table 2: Textures with corresponding skeleton primitive weights by using MHDWT

\begin{tabular}{lll}
\hline Texture & $\begin{array}{l}\text { No. of } \\
\text { skeleton points }\end{array}$ & $\begin{array}{l}\text { Dominant skeleton } \\
\text { primitive weight }\end{array}$ \\
\hline $\mathrm{D}_{1}$ & 5463 & 255 \\
$\mathrm{D}_{2}$ & 4684 & 255 \\
$\mathrm{D}_{3}$ & 8201 & 255 \\
$\mathrm{D}_{4}$ & 7698 & 251 \\
$\mathrm{D}_{5}$ & 4772 & 255 \\
$\mathrm{D}_{6}$ & 5469 & 153 \\
$\mathrm{D}_{7}$ & 3111 & 255 \\
$\mathrm{D}_{8}$ & 2167 & 255 \\
$\mathrm{D}_{9}$ & 7216 & 255 \\
$\mathrm{D}_{10}$ & 6371 & 255 \\
$\mathrm{D}_{11}$ & 6181 & 255 \\
$\mathrm{D}_{12}$ & 5715 & 255 \\
$\mathrm{D}_{13}$ & 4545 & 255 \\
$\mathrm{D}_{15}$ & 6868 & 255 \\
$\mathrm{D}_{16}$ & 6128 & 255 \\
$\mathrm{D}_{17}$ & 6898 & 54 \\
$\mathrm{D}_{18}$ & 4177 & 255 \\
$\mathrm{D}_{19}$ & 5763 & 255 \\
$\mathrm{D}_{20}$ & 4683 & 255 \\
$\mathrm{D}_{21}$ & 6873 & 112 \\
$\mathrm{D}_{22}$ & 7093 & 255 \\
$\mathrm{D}_{23}$ & 3524 & 255 \\
$\mathrm{D}_{24}$ & 8087 & 255 \\
$\mathrm{D}_{25}$ & 2171 & 255 \\
\hline
\end{tabular}

Table 3: Textures with corresponding skeleton primitive weights by using MBWT

\begin{tabular}{lll}
\hline & $\begin{array}{l}\text { No. of } \\
\text { skeleton points }\end{array}$ & $\begin{array}{l}\text { Dominant skeleton } \\
\text { primitive weight }\end{array}$ \\
\hline $\mathrm{D}_{1}$ & 4221 & 255 \\
$\mathrm{D}_{2}$ & 4542 & 255 \\
$\mathrm{D}_{3}$ & 8244 & 255 \\
$\mathrm{D}_{4}$ & 7591 & 223 \\
$\mathrm{D}_{5}$ & 4670 & 255 \\
$\mathrm{D}_{6}$ & 4452 & 153 \\
$\mathrm{D}_{7}$ & 3584 & 255 \\
$\mathrm{D}_{8}$ & 2150 & 255 \\
$\mathrm{D}_{9}$ & 6922 & 255 \\
$\mathrm{D}_{10}$ & 5811 & 255 \\
$\mathrm{D}_{11}$ & 6398 & 255 \\
$\mathrm{D}_{12}$ & 5460 & 255 \\
$\mathrm{D}_{13}$ & 4267 & 255 \\
$\mathrm{D}_{15}$ & 6988 & 255 \\
$\mathrm{D}_{16}$ & 5883 & 255 \\
$\mathrm{D}_{17}$ & 6746 & 109 \\
$\mathrm{D}_{18}$ & 4032 & 255 \\
$\mathrm{D}_{19}$ & 5717 & 255 \\
$\mathrm{D}_{20}$ & 3568 & 255 \\
$\mathrm{D}_{21}$ & 4125 & 24 \\
$\mathrm{D}_{22}$ & 6795 & 206 \\
$\mathrm{D}_{23}$ & 3512 & 255 \\
$\mathrm{D}_{24}$ & 7920 & 115 \\
$\mathrm{D}_{25}$ & 2687 & 255 \\
\hline & &
\end{tabular}

MHEWC $_{1}=\left\{D_{1}, D_{2}, D_{5}, D_{7}, D_{8}, D_{9}, D_{10}, D_{11}, D_{12}, D_{13}\right.$, $\left.\mathrm{D}_{15}, \mathrm{D}_{16}, \mathrm{D}_{18}, \mathrm{D}_{19}, \mathrm{D}_{20}, \mathrm{D}_{23}, \mathrm{D}_{25}\right\}$

$\mathrm{MHDWC}_{1}=\left\{\mathrm{D}_{1}, \mathrm{D}_{2}, \mathrm{D}_{3}, \mathrm{D}_{5}, \mathrm{D}_{7}, \mathrm{D}_{8}, \mathrm{D}_{9}, \mathrm{D}_{10}, \mathrm{D}_{11}, \mathrm{D}_{12}\right.$, $\mathrm{D}_{13}, \mathrm{D}_{15}, \mathrm{D}_{16}, \mathrm{D}_{18}, \mathrm{D}_{19}, \mathrm{D}_{20}, \mathrm{D}_{22}, \mathrm{D}_{23}, \mathrm{D}_{24}$, , $\left.\mathrm{D}_{25}\right\}$ 
$\begin{aligned} \text { MBWC }_{1}= & \left\{\mathrm{D}_{1}, \mathrm{D}_{2}, \mathrm{D}_{3}, \mathrm{D} 5, \mathrm{D}_{7}, \mathrm{D}_{8}, \mathrm{D}_{9}, \mathrm{D}_{10}, \mathrm{D}_{11}, \mathrm{D}_{12},\right. \\ & \left.\mathrm{D}_{13}, \mathrm{D}_{15}, \mathrm{D}_{16}, \mathrm{D}_{18}, \mathrm{D}_{19}, \mathrm{D}_{20}, \mathrm{D}_{23}, \mathrm{D}_{25}\right\}\end{aligned}$

Out of these, 17 textures are having a dominant skeleton primitive with a weight of 255 for all three nonlinear wavelet transforms considered and they are listed below.

$\left\{D_{1}, D_{2}, D_{5}, D_{7}, D_{8}, D_{9}, D_{10}, D_{11}, D_{12}, D_{13}, D_{15}, D_{16}\right.$, $\left.\mathrm{D}_{18}, \mathrm{D}_{19}, \mathrm{D}_{20}, \mathrm{D}_{23}, \mathrm{D}_{25}\right\}$

And no other texture is having any other skeleton primitive with common weight in all three nonlinear wavelet transforms: MHEW, MHDW and MBW. By this, the present study evaluates a common classification rate based on skeleton primitives as $70.83 \%$. Further the present study classified textures based on their least number of skeleton points using distance function as follows. By distance function ${ }^{[29]}$ we say that two textures are similar if and only if they contain the same number of primitive patterns or same percentage of occurrence of patterns as given in Eq. 1.

$$
D(i)=\sum_{j=1}^{p} \operatorname{abs}\left(F_{j}(x)-F_{j}(i)\right)=0
$$

where $\mathrm{p}$ stands for the total number of features used, $\mathrm{i}=$ 1 to $Q$ ( $Q$ is the number of classes in the database), $f_{j}(x)$, represents the $j^{\text {th }}$ feature of unknown texture class (x) and $\mathrm{f}_{\mathrm{j}}(\mathrm{i})$ represents the $\mathrm{j}^{\text {th }}$ feature of texture belonging to $i^{\text {th }}$ class.

Equation 1 is a rare phenomenon for classification of textures based on skeleton points, because they appear in large number. To avoid this, a lag value based distance function is given in the Eq. 2 .

$$
D(i)=\sum_{j=1}^{p} \operatorname{abs}\left(F_{j}(x)-F_{j}(i)\right) \leq 1_{i}
$$

Equation 2 is modified in the present study as:

$$
\mathrm{D}\left(\mathrm{WT}_{\mathrm{i}}, \mathrm{WT}_{\mathrm{j}}\right)=\left|\mathrm{NSP}_{\mathrm{i}}-\mathrm{NSP}_{\mathrm{j}}\right| \leq 1_{\mathrm{i}}
$$

where $\mathrm{WT}_{\mathrm{i}}$ and $\mathrm{WT}_{\mathrm{j}}$ are the wavelet transformed Brodatz textures , $1 \leq \mathrm{i}, \mathrm{j} \leq 24, \mathrm{NSP}_{\mathrm{i}}$ and $\mathrm{NSP}_{\mathrm{j}}$ are the number of skeleton points in the Skeletons of the two textures. This Eq. 3 suggests that two textures are similar if their distances or percentage of occurrence is less than or equal to lag value ' $\mathrm{l}_{\mathrm{i}}$ '. This indicates that lag value plays crucial role and by increasing lag value the percentage of correct classification (PCC) increases. The distances among all 24 textures based on number of skeleton points using MHE, MHD and MB wavelet transform are listed in Table 4, 5 and 6 respectively. Based on the lag value a classification is resulted in the following way. That is two or more textures belong to the same class $C_{i}$ if they differ with the lag value less

\begin{tabular}{|c|c|c|c|c|c|c|c|c|c|c|c|c|c|c|c|c|c|c|c|c|c|c|c|c|}
\hline & $\mathrm{D}_{1}$ & $\mathrm{D}_{2}$ & $\mathrm{D}_{3}$ & $\mathrm{D}_{4}$ & $\mathrm{D}_{5}$ & $\mathrm{D}_{6}$ & $\mathrm{D}_{7}$ & $\mathrm{D}_{8}$ & $\mathrm{D}_{9}$ & $\mathrm{D}_{10}$ & $\mathrm{D}_{11}$ & $\mathrm{D}_{12}$ & $\mathrm{D}_{13}$ & $\mathrm{D}_{15}$ & $\mathrm{D}_{16}$ & $\mathrm{D}_{17}$ & $\mathrm{D}_{18}$ & $\mathrm{D}_{19}$ & $\mathrm{D}_{20}$ & $\mathrm{D}_{21}$ & $\mathrm{D}_{22}$ & $\mathrm{D}_{23}$ & $\mathrm{D}_{24}$ & $\mathrm{D}_{25}$ \\
\hline$\overline{\mathrm{D}_{1}}$ & 0 & 18 & 54 & 57 & 22 & 6 & 15 & 41 & 47 & 41 & 49 & 24 & 9 & 46 & 61 & 60 & 11 & 37 & 35 & 31 & 38 & 23 & 55 & 6 \\
\hline $\mathrm{D}_{2}$ & 18 & 0 & 51 & 54 & 13 & 19 & 23 & 45 & 44 & 37 & 46 & 16 & 20 & 42 & 58 & 57 & 21 & 32 & 40 & 36 & 33 & 29 & 52 & 17 \\
\hline $\mathrm{D}_{3}$ & 54 & 51 & 0 & 17 & 50 & 55 & 56 & 68 & 26 & 36 & 22 & 48 & 55 & 29 & 28 & 26 & 55 & 39 & 65 & 63 & 39 & 59 & 10 & 54 \\
\hline $\mathrm{D}_{4}$ & 57 & 54 & 17 & 0 & 52 & 57 & 59 & 70 & 31 & 39 & 28 & 51 & 57 & 34 & 22 & 20 & 58 & 43 & 67 & 65 & 42 & 61 & 13 & 56 \\
\hline $\mathrm{D}_{5}$ & 22 & 13 & 50 & 52 & 0 & 23 & 27 & 47 & 42 & 34 & 44 & 10 & 24 & 40 & 57 & 56 & 25 & 30 & 42 & 38 & 31 & 32 & 51 & 21 \\
\hline $\mathrm{D}_{6}$ & 6 & 19 & 55 & 57 & 23 & 0 & 14 & 41 & 48 & 41 & 50 & 25 & 7 & 46 & 61 & 60 & 9 & 38 & 35 & 30 & 38 & 22 & 56 & 9 \\
\hline $\mathrm{D}_{7}$ & 15 & 23 & 56 & 59 & 27 & 14 & 0 & 39 & 50 & 43 & 52 & 29 & 12 & 48 & 63 & 62 & 10 & 40 & 32 & 27 & 41 & 17 & 57 & 16 \\
\hline $\mathrm{D}_{8}$ & 41 & 45 & 68 & 70 & 47 & 41 & 39 & 0 & 63 & 58 & 64 & 48 & 40 & 62 & 74 & 73 & 40 & 56 & 22 & 27 & 56 & 35 & 69 & 42 \\
\hline $\mathrm{D}_{9}$ & 47 & 44 & 26 & 31 & 42 & 48 & 50 & 63 & 0 & 24 & 14 & 41 & 48 & 13 & 38 & 37 & 49 & 29 & 59 & 57 & 28 & 52 & 28 & 47 \\
\hline $\mathrm{D}_{10}$ & 41 & 37 & 36 & 39 & 34 & 41 & 43 & 58 & 24 & 0 & 28 & 33 & 42 & 20 & 45 & 44 & 42 & 17 & 54 & 51 & 15 & 47 & 37 & 40 \\
\hline $\mathrm{D}_{11}$ & 49 & 46 & 22 & 28 & 44 & 50 & 52 & 64 & 14 & 28 & 0 & 43 & 50 & 19 & 36 & 34 & 51 & 33 & 61 & 58 & 32 & 54 & 25 & 49 \\
\hline $\mathrm{D}_{12}$ & 24 & 16 & 48 & 51 & 10 & 25 & 29 & 48 & 41 & 33 & 43 & 0 & 26 & 39 & 56 & 55 & 27 & 28 & 43 & 39 & 29 & 33 & 50 & 23 \\
\hline $\mathrm{D}_{13}$ & 9 & 20 & 55 & 57 & 24 & 7 & 12 & 40 & 48 & 42 & 50 & 26 & 0 & 47 & 62 & 61 & 5 & 38 & 34 & 30 & 39 & 21 & 56 & 11 \\
\hline $\mathrm{D}_{15}$ & 46 & 42 & 29 & 34 & 40 & 46 & 48 & 62 & 13 & 20 & 19 & 39 & 47 & 0 & 40 & 39 & 47 & 26 & 58 & 55 & 25 & 51 & 31 & 45 \\
\hline $\mathrm{D}_{16}$ & 61 & 58 & 28 & 22 & 57 & 61 & 63 & 74 & 38 & 45 & 36 & 56 & 62 & 40 & 0 & 10 & 62 & 48 & 70 & 68 & 48 & 65 & 26 & 61 \\
\hline $\mathrm{D}_{17}$ & 60 & 57 & 26 & 20 & 56 & 60 & 62 & 73 & 37 & 44 & 34 & 55 & 61 & 39 & 10 & 0 & 61 & 47 & 70 & 68 & 47 & 64 & 24 & 60 \\
\hline $\mathrm{D}_{18}$ & 11 & 21 & 55 & 58 & 25 & 9 & 10 & 40 & 49 & 42 & 51 & 27 & 5 & 47 & 62 & 61 & 0 & 39 & 34 & 29 & 40 & 20 & 56 & 13 \\
\hline $\mathrm{D}_{19}$ & 37 & 32 & 39 & 43 & 30 & 38 & 40 & 56 & 29 & 17 & 33 & 28 & 38 & 26 & 48 & 47 & 39 & 0 & 51 & 48 & 8 & 43 & 41 & 37 \\
\hline $\mathrm{D}_{20}$ & 35 & 40 & 65 & 67 & 42 & 35 & 32 & 22 & 59 & 54 & 61 & 43 & 34 & 58 & 70 & 70 & 34 & 51 & 0 & 17 & 52 & 27 & 66 & 36 \\
\hline $\mathrm{D}_{21}$ & 31 & 36 & 63 & 65 & 38 & 30 & 27 & 27 & 57 & 51 & 58 & 39 & 30 & 55 & 68 & 68 & 29 & 48 & 17 & 0 & 49 & 21 & 63 & 32 \\
\hline $\mathrm{D}_{22}$ & 38 & 33 & 39 & 42 & 31 & 38 & 41 & 56 & 28 & 15 & 32 & 29 & 39 & 25 & 48 & 47 & 40 & 8 & 52 & 49 & 0 & 44 & 40 & 37 \\
\hline $\mathrm{D}_{23}$ & 23 & 29 & 59 & 61 & 32 & 22 & 17 & 35 & 52 & 47 & 54 & 33 & 21 & 51 & 65 & 64 & 20 & 43 & 27 & 21 & 44 & 0 & 60 & 23 \\
\hline $\mathrm{D}_{24}$ & 55 & 52 & 10 & 13 & 51 & 56 & 57 & 69 & 28 & 37 & 25 & 50 & 56 & 31 & 26 & 24 & 56 & 41 & 66 & 63 & 40 & 60 & 0 & 55 \\
\hline $\mathrm{D}_{25}$ & 6 & 17 & 54 & 56 & 21 & 9 & 16 & 42 & 47 & 40 & 49 & 23 & 11 & 45 & 61 & 60 & 13 & 37 & 36 & 32 & 37 & 23 & 55 & 0 \\
\hline
\end{tabular}
than or equal to $l_{i}$.

Table 4: Distance among the textures based on number of skeleton points using MHE Wavelet Transform 


\section{J. Computer Sci., 4 (7): 591-599, 2008}

Table 5: Distance among the textures based on number of skeleton points using MHD Wavelet Transform

\begin{tabular}{|c|c|c|c|c|c|c|c|c|c|c|c|c|c|c|c|c|c|c|c|c|c|c|c|c|}
\hline & $D_{1}$ & $\mathrm{D}_{2}$ & $\mathrm{D}_{3}$ & $\mathrm{D}_{4}$ & $D_{5}$ & $D_{6}$ & $\mathrm{D}_{7}$ & $\mathrm{D}_{8}$ & $\mathrm{D}_{9}$ & $\mathrm{D}_{10}$ & $\mathrm{D}_{11}$ & $\mathrm{D}_{12}$ & $\mathrm{D}_{13}$ & $\mathrm{D}_{15}$ & $\mathrm{D}_{16}$ & $\mathrm{D}_{17}$ & $\mathrm{D}_{18}$ & $\mathrm{D}_{19}$ & $\mathrm{D}_{20}$ & $\mathrm{D}_{21}$ & $\mathrm{D}_{22}$ & $\mathrm{D}_{23}$ & $\mathrm{D}_{24}$ & $\mathrm{D}_{25}$ \\
\hline$\overline{D_{1}}$ & 0 & 28 & 52 & 47 & 26 & 2 & 48 & 57 & 42 & 30 & 27 & 16 & 30 & 37 & 26 & 38 & 36 & 17 & 28 & 38 & 40 & 44 & 51 & 57 \\
\hline$D_{2}$ & 28 & 0 & 59 & 55 & 9 & 28 & 40 & 50 & 50 & 41 & 39 & 32 & 12 & 47 & 38 & 47 & 23 & 33 & 1 & 47 & 49 & 34 & 58 & 50 \\
\hline $\mathrm{D}_{3}$ & 52 & 59 & 0 & 22 & 59 & 52 & 71 & 78 & 31 & 43 & 45 & 50 & 60 & 37 & 46 & 36 & 63 & 49 & 59 & 36 & 33 & 68 & 11 & 78 \\
\hline $\mathrm{D}_{4}$ & 47 & 55 & 22 & 0 & 54 & 47 & 68 & 74 & 22 & 36 & 39 & 45 & 56 & 29 & 40 & 28 & 59 & 44 & 55 & 29 & 25 & 65 & 20 & 74 \\
\hline$D_{5}$ & 26 & 9 & 59 & 54 & 0 & 26 & 41 & 51 & 49 & 40 & 38 & 31 & 15 & 46 & 37 & 46 & 24 & 31 & 9 & 46 & 48 & 35 & 58 & 51 \\
\hline$D_{6}$ & 2 & 28 & 52 & 47 & 26 & 0 & 49 & 57 & 42 & 30 & 27 & 16 & 30 & 37 & 26 & 38 & 36 & 17 & 28 & 37 & 40 & 44 & 51 & 57 \\
\hline $\mathrm{D}_{7}$ & 48 & 40 & 71 & 68 & 41 & 49 & 0 & 31 & 64 & 57 & 55 & 51 & 38 & 61 & 55 & 62 & 33 & 51 & 40 & 61 & 63 & 20 & 71 & 31 \\
\hline $\mathrm{D}_{8}$ & 57 & 50 & 78 & 74 & 51 & 57 & 31 & 0 & 71 & 65 & 63 & 60 & 49 & 69 & 63 & 69 & 45 & 60 & 50 & 69 & 70 & 37 & 77 & 2 \\
\hline $\mathrm{D}_{9}$ & 42 & 50 & 31 & 22 & 49 & 42 & 64 & 71 & 0 & 29 & 32 & 39 & 52 & 19 & 33 & 18 & 55 & 38 & 50 & 19 & 11 & 61 & 30 & 71 \\
\hline$D_{10}$ & 30 & 41 & 43 & 36 & 40 & 30 & 57 & 65 & 29 & 0 & 14 & 26 & 43 & 22 & 16 & 23 & 47 & 25 & 41 & 22 & 27 & 53 & 41 & 65 \\
\hline $\mathrm{D}_{11}$ & 27 & 39 & 45 & 39 & 38 & 27 & 55 & 63 & 32 & 14 & 0 & 22 & 40 & 26 & 7 & 27 & 45 & 20 & 39 & 26 & 30 & 52 & 44 & 63 \\
\hline$D_{12}$ & 16 & 32 & 50 & 45 & 31 & 16 & 51 & 60 & 39 & 26 & 22 & 0 & 34 & 34 & 20 & 34 & 39 & 7 & 32 & 34 & 37 & 47 & 49 & 60 \\
\hline$D_{13}$ & 30 & 12 & 60 & 56 & 15 & 30 & 38 & 49 & 52 & 43 & 40 & 34 & 0 & 48 & 40 & 49 & 19 & 35 & 12 & 48 & 50 & 32 & 60 & 49 \\
\hline $\mathrm{D}_{15}$ & 37 & 47 & 37 & 29 & 46 & 37 & 61 & 69 & 19 & 22 & 26 & 34 & 48 & 0 & 27 & 5 & 52 & 33 & 47 & 2 & 15 & 58 & 35 & 69 \\
\hline $\mathrm{D}_{16}$ & 26 & 38 & 46 & 40 & 37 & 26 & 55 & 63 & 33 & 16 & 7 & 20 & 40 & 27 & 0 & 28 & 44 & 19 & 38 & 27 & 31 & 51 & 44 & 63 \\
\hline$D_{17}$ & 38 & 47 & 36 & 28 & 46 & 38 & 62 & 69 & 18 & 23 & 27 & 34 & 49 & 5 & 28 & 0 & 52 & 34 & 47 & 5 & 14 & 58 & 34 & 69 \\
\hline $\mathrm{D}_{18}$ & 36 & 23 & 63 & 59 & 24 & 36 & 33 & 45 & 55 & 47 & 45 & 39 & 19 & 52 & 44 & 52 & 0 & 40 & 22 & 52 & 54 & 26 & 63 & 45 \\
\hline $\mathrm{D}_{19}$ & 17 & 33 & 49 & 44 & 31 & 17 & 51 & 60 & 38 & 25 & 20 & 7 & 35 & 33 & 19 & 34 & 40 & 0 & 33 & 33 & 36 & 47 & 48 & 60 \\
\hline $\mathrm{D}_{20}$ & 28 & 1 & 59 & 55 & 9 & 28 & 40 & 50 & 50 & 41 & 39 & 32 & 12 & 47 & 38 & 47 & 22 & 33 & 0 & 47 & 49 & 34 & 58 & 50 \\
\hline $\mathrm{D}_{21}$ & 38 & 47 & 36 & 29 & 46 & 37 & 61 & 69 & 19 & 22 & 26 & 34 & 48 & 2 & 27 & 5 & 52 & 33 & 47 & 0 & 15 & 58 & 35 & 69 \\
\hline $\mathrm{D}_{22}$ & 40 & 49 & 33 & 25 & 48 & 40 & 63 & 70 & 11 & 27 & 30 & 37 & 50 & 15 & 31 & 14 & 54 & 36 & 49 & 15 & 0 & 60 & 32 & 70 \\
\hline$D_{23}$ & 44 & 34 & 68 & 65 & 35 & 44 & 20 & 37 & 61 & 53 & 52 & 47 & 32 & 58 & 51 & 58 & 26 & 47 & 34 & 58 & 60 & 0 & 68 & 37 \\
\hline$D_{24}$ & 51 & 58 & 11 & 20 & 58 & 51 & 71 & 77 & 30 & 41 & 44 & 49 & 60 & 35 & 44 & 34 & 63 & 48 & 58 & 35 & 32 & 68 & 0 & 77 \\
\hline$D_{25}$ & 57 & 50 & 78 & 74 & 51 & 57 & 31 & 2 & 71 & 65 & 63 & 60 & 49 & 69 & 63 & 69 & 45 & 60 & 50 & 69 & 70 & 37 & 77 & 0 \\
\hline
\end{tabular}

Table 6: Distance among the textures based on number of skeleton points using MB Wavelet Transform

\begin{tabular}{|c|c|c|c|c|c|c|c|c|c|c|c|c|c|c|c|c|c|c|c|c|c|c|c|c|}
\hline & $D_{1}$ & $D_{2}$ & $D_{3}$ & $\mathrm{D}_{4}$ & $D_{5}$ & $D_{6}$ & $D_{7}$ & $D_{8}$ & $\mathrm{D}_{9}$ & $\mathrm{D}_{10}$ & $\mathrm{D}_{11}$ & $D_{12}$ & $D_{13}$ & $D_{15}$ & $D_{16}$ & $D_{17}$ & $D_{18}$ & $D_{19}$ & $\mathrm{D}_{20}$ & $D_{21}$ & $\mathrm{D}_{22}$ & $D_{23}$ & $\mathrm{D}_{24}$ & $\mathrm{D}_{25}$ \\
\hline$D_{1}$ & 0 & 18 & 63 & 58 & 21 & 15 & 25 & 46 & 52 & 40 & 47 & 35 & 7 & 53 & 41 & 50 & 14 & 39 & 26 & 10 & 51 & 27 & 61 & 39 \\
\hline$D_{2}$ & 18 & 0 & 61 & 55 & 11 & 9 & 31 & 49 & 49 & 36 & 43 & 30 & & 49 & & & & 34 & & & & 32 & 58 & 43 \\
\hline $\mathrm{D}_{3}$ & 63 & 61 & 0 & 26 & 60 & 62 & 68 & 78 & 36 & 49 & 43 & 53 & & & & & & 50 & & & & 69 & 18 & 75 \\
\hline$D_{4}$ & 58 & 55 & 26 & 0 & 54 & 56 & 63 & 72 & 26 & 42 & 35 & 46 & & 25 & 41 & 29 & 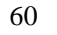 & 43 & 63 & 5 & 28 & 64 & 18 & 70 \\
\hline$)_{5}$ & 21 & 11 & 60 & 5 & 0 & 15 & 33 & 5 & 47 & 34 & 42 & 28 & & 4 & & 4 & & 32 & 33 & 23 & 46 & 34 & 57 & 45 \\
\hline 6 & 15 & 9 & 6 & 5 & 15 & 0 & 29 & 4 & 50 & 3 & 4 & 32 & & & & & & 36 & & & 8 & 31 & 59 & 42 \\
\hline & 2 & 31 & & & 3. & 29 & 0 & & 58 & 4 & 5 & 4 & & & & & & 46 & & & & & 66 & 30 \\
\hline & 46 & 49 & 78 & 7 & 50 & 48 & 38 & 0 & 69 & 6 & 65 & 58 & 4 & 70 & 6 & 68 & 43 & 60 & 38 & 4 & & 37 & 76 & 23 \\
\hline$\rho_{9}$ & 5 & 49 & 36 & 26 & 47 & 50 & 58 & 69 & & 3. & 2. & 38 & & & & 1. & & 3. & Jo & 5. & & 58 & 32 & 65 \\
\hline$D_{10}$ & & 36 & 4 & 4 & 34 & 37 & 47 & 6 & 33 & & 2 & 19 & & 34 & & & & 10 & & 4 & & 48 & 46 & 56 \\
\hline$D_{1}$ & 4 & 43 & 43 & 35 & 42 & 44 & 53 & 6 & 23 & 24 & 0 & 31 & & 24 & 23 & 1 & 49 & 26 & 53 & 4 & & 54 & 39 & 61 \\
\hline$D_{1}$ & 35 & 30 & 5 & 46 & 28 & 32 & 43 & & 38 & 1 & 31 & 0 & & 39 & 21 & & & 16 & 43 & & & 44 & 50 & 53 \\
\hline & 7 & 17 & & & 20 & 14 & 26 & 4 & 5 & 3 & 4 & & & & & & & 38 & & & & 27 & 60 & 40 \\
\hline & 5 & 49 & & 2 & 48 & 50 & 58 & 70 & & 3 & 2 & 39 & & & & & & 36 & & & & 59 & 31 & 66 \\
\hline $\mathrm{D}_{16}$ & 41 & 37 & 4 & 4 & 35 & 38 & 48 & 6 & 32 & 8 & 2 & 21 & & 33 & & 29 & 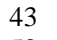 & 13 & 48 & 4 & 30 & 49 & 45 & 57 \\
\hline$D_{17}$ & 5 & 47 & 36 & 2 & 46 & 48 & 5 & & 13 & 31 & 19 & 36 & 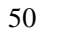 & 16 & 2 & & 5 & 32 & 58 & 5 & & 57 & 34 & 64 \\
\hline $\mathrm{D}_{18}$ & 1 & 23 & 6 & & 2 & 20 & 2 & 4 & 5 & 4 & 4 & 3 & & 5 & 4 & & & 41 & 2 & & & 23 & 62 & 37 \\
\hline $\mathrm{D}_{19}$ & 39 & 34 & 50 & 4 & 32 & 36 & 46 & 60 & 3 & 10 & 26 & 16 & & 3 & 1 & 3 & 4 & & 46 & & & 47 & 47 & 55 \\
\hline $\mathrm{D}_{20}$ & 26 & 31 & 68 & 6 & 33 & 30 & 4 & 3 & 58 & 47 & 53 & 4 & & & & & & 46 & & & & & 66 & 30 \\
\hline & 10 & 20 & & & 2 & 18 & & & & 41 & & & & & & & & & & & & & 62 & 38 \\
\hline & 5 & 47 & 3 & 2 & 46 & 48 & & & & 3 & 2 & 3 & & & 3 & & & 3 & & & & 57 & 34 & 64 \\
\hline $\mathrm{D}$ & 2 & 32 & 6 & & 3 & 31 & $\varepsilon$ & & & $4 \varepsilon$ & & 4 & & & 4 & & & 47 & & & & & 66 & 29 \\
\hline$D_{2}$ & & 58 & 1 & & 5 & 59 & & & & 46 & 39 & 5 & & 3 & 4 & & & 47 & 6 & & & 66 & 0 & 72 \\
\hline $\mathrm{D}_{25}$ & 39 & 43 & 75 & 70 & 45 & 42 & 30 & 23 & 65 & 56 & 61 & 53 & 40 & 66 & 57 & 64 & 37 & 55 & 30 & 38 & 64 & 29 & 72 & 0 \\
\hline
\end{tabular}

When lag value is 30 the classes resulted based on least number of skeleton primitive points for the three nonlinear wavelets are shown in Table 7 . In the same way, when the lag value is 50 the resultant classes are shown in Table 8.

If we assume MB wavelet transform as standard one then the classification rates are evaluated for each class with other nonlinear wavelets and they are listed in Table 9 and Table 10 for lag value 30 and 50 respectively. The classification rates are evaluated by the following formula: Percentage of classification rate $=$ Number of common textures of $\mathrm{W}_{\mathrm{x}}$ with respect to $\mathrm{W}_{\mathrm{y}}$ for class $\mathrm{Ci} /$ Number of textures in $\mathrm{Wy}$ for class $\mathrm{C}_{\mathrm{i}}$ that means if $\mathrm{W}_{\mathrm{y}}$ has got ' $\mathrm{l}$ ' textures in class $\mathrm{C}_{\mathrm{i}}$ and $\mathrm{W}_{\mathrm{x}}$ has got ' $1+m$ ' textures then by the above formula shows $100 \%$ of correct classification for class $C_{i}$. However this effects for another class because of extra ' $\mathrm{m}$ ' textures. By this the above formula guarantees that overall texture classification rate does not change and comparison rate is not going to be effected. 
Table 7: Classes of textures for the three nonlinear wavelets when the

\begin{tabular}{lll}
\multicolumn{2}{c}{ lag value is 30} & \\
\hline Wavelet & Class & Textures \\
\hline MHEWT & $\mathrm{C}_{1}$ & $\left\{\mathrm{D}_{1}, \mathrm{D}_{2}, \mathrm{D}_{5}, \mathrm{D}_{6}, \mathrm{D}_{7}, \mathrm{D}_{12}, \mathrm{D}_{13}, \mathrm{D}_{18}, \mathrm{D}_{25}\right\}$ \\
& $\mathrm{C}_{2}$ & $\left\{\mathrm{D}_{3}, \mathrm{D}_{4}, \mathrm{D}_{11}, \mathrm{D}_{24}\right\}$ \\
& $\mathrm{C}_{3}$ & $\left\{\mathrm{D}_{8}, \mathrm{D}_{20}, \mathrm{D}_{21}\right\}$ \\
& $\mathrm{C}_{4}$ & $\left\{\mathrm{D}_{9}, \mathrm{D}_{10}, \mathrm{D}_{15}, \mathrm{D}_{19}, \mathrm{D}_{22}\right\}$ \\
& $\mathrm{C}_{5}$ & $\left\{\mathrm{D}_{16}, \mathrm{D}_{17}\right\}$ \\
& $\mathrm{C}_{6}$ & $\left\{\mathrm{D}_{23}\right\}$ \\
MHDWT & $\mathrm{C}_{1}$ & $\left\{\mathrm{D}_{1}, \mathrm{D}_{2}, \mathrm{D}_{5}, \mathrm{D}_{6}, \mathrm{D}_{13}, \mathrm{D}_{20}\right\}$ \\
& $\mathrm{C}_{2}$ & $\left\{\mathrm{D}_{3}, \mathrm{D}_{4}, \mathrm{D}_{24}\right\}$ \\
& $\mathrm{C}_{3}$ & $\left\{\mathrm{D}_{7}, \mathrm{D}_{23}\right\}$ \\
& $\mathrm{C}_{4}$ & $\left\{\mathrm{D}_{8}, \mathrm{D}_{25}\right\}$ \\
& $\mathrm{C}_{5}$ & $\left\{\mathrm{D}_{9}, \mathrm{D}_{10}, \mathrm{D}_{15}, \mathrm{D}_{17}, \mathrm{D}_{21}, \mathrm{D}_{22}\right\}$ \\
& $\mathrm{C}_{6}$ & $\left\{\mathrm{D}_{11}, \mathrm{D}_{12}, \mathrm{D}_{16}, \mathrm{D}_{19}\right\}$ \\
& $\mathrm{C}_{7}$ & $\left\{\mathrm{D}_{18}\right\}$ \\
& $\mathrm{C}_{1}$ & $\left\{\mathrm{D}_{1}, \mathrm{D}_{2}, \mathrm{D}_{5}, \mathrm{D}_{6}, \mathrm{D}_{13}, \mathrm{D}_{18}, \mathrm{D}_{21}\right\}$ \\
& $\mathrm{C}_{2}$ & $\left\{\mathrm{D}_{3}, \mathrm{D}_{4}, \mathrm{D}_{24}\right\}$ \\
& $\mathrm{C}_{3}$ & $\left\{\mathrm{D}_{7}, \mathrm{D}_{20}, \mathrm{D}_{23}, \mathrm{D}_{25}\right\}$ \\
& $\mathrm{C}_{4}$ & $\left\{\mathrm{D}_{8}\right\}$ \\
& $\mathrm{C}_{5}$ & $\left\{\mathrm{D}_{9}, \mathrm{D}_{11}, \mathrm{D}_{15}, \mathrm{D}_{17}, \mathrm{D}_{22}\right\}$ \\
& $\mathrm{C}_{6}$ & $\left\{\mathrm{D}_{10}, \mathrm{D}_{12}, \mathrm{D}_{16}, \mathrm{D}_{19}\right\}$ \\
& &
\end{tabular}

Table 8: Classes of textures for the three nonlinear wavelets when the lag value is 50

\begin{tabular}{lll}
\hline Wavelet & Class & Textures \\
\hline MHEWT & $\mathrm{C}_{1}$ & $\left\{\mathrm{D}_{1}, \mathrm{D}_{2}, \mathrm{D}_{5}, \mathrm{D}_{6}, \mathrm{D}_{7}, \mathrm{D}_{8}, \mathrm{D}_{12}, \mathrm{D}_{13}, \mathrm{D}_{18}, \mathrm{D}_{20}, \mathrm{D}_{21}, \mathrm{D}_{23}, \mathrm{D}_{25}\right\}$ \\
& $\mathrm{C}_{2}$ & $\left\{\mathrm{D}_{3}, \mathrm{D}_{4}, \mathrm{D}_{9}, \mathrm{D}_{10}, \mathrm{D}_{11}, \mathrm{D}_{15}, \mathrm{D}_{16}, \mathrm{D}_{17}, \mathrm{D}_{19}, \mathrm{D}_{22}, \mathrm{D}_{24}\right\}$ \\
MHDWT & $\mathrm{C}_{1}$ & $\left\{\mathrm{D}_{1}, \mathrm{D}_{2}, \mathrm{D}_{5}, \mathrm{D}_{6}, \mathrm{D}_{7}, \mathrm{D}_{13}, \mathrm{D}_{18}, \mathrm{D}_{20}, \mathrm{D}_{23}\right\}$ \\
& $\mathrm{C}_{2}$ & $\left\{\mathrm{D}_{3}, \mathrm{D}_{4}, \mathrm{D}_{9}, \mathrm{D}_{10}, \mathrm{D}_{11}, \mathrm{D}_{12}, \mathrm{D}_{15}, \mathrm{D}_{16}, \mathrm{D}_{17}, \mathrm{D}_{19}, \mathrm{D}_{21}, \mathrm{D}_{22}, \mathrm{D}_{24}\right\}$ \\
& $\mathrm{C}_{3}$ & $\left\{\mathrm{D}_{8}, \mathrm{D}_{25}\right\}$ \\
MBWT & $\mathrm{C}_{1}$ & $\left\{\mathrm{D}_{1}, \mathrm{D}_{2}, \mathrm{D}_{5}, \mathrm{D}_{6}, \mathrm{D}_{7}, \mathrm{D}_{8}, \mathrm{D}_{13}, \mathrm{D}_{18}, \mathrm{D}_{20}, \mathrm{D}_{21}, \mathrm{D}_{23}, \mathrm{D}_{25}\right\}$ \\
& $\mathrm{C}_{2}$ & $\left\{\mathrm{D}_{3}, \mathrm{D}_{4}, \mathrm{D}_{9}, \mathrm{D}_{10}, \mathrm{D}_{11}, \mathrm{D}_{15}, \mathrm{D}_{16}, \mathrm{D}_{17}, \mathrm{D}_{19}, \mathrm{D}_{22}, \mathrm{D}_{24}\right\}$ \\
& $\mathrm{C}_{3}$ & $\left\{\mathrm{D}_{12}\right\}$ \\
\hline
\end{tabular}

Table 9: Classification rates of MHEWT and MHDWT with respect to MBWT when lag value $=30$

\begin{tabular}{lll}
\hline Class & MHEWT & MHDWT \\
\hline 1 & 85.71 & 71.43 \\
2 & 100.00 & 100.00 \\
3 & 25.00 & 50.00 \\
4 & 0.00 & 100.00 \\
5 & 20.00 & 80.00 \\
6 & 0.00 & 75.00 \\
Average & 38.45 & 79.41 \\
\hline
\end{tabular}

Table 10: Classification rates of MHEWT and MHDWT with respect to MBWT when lag value $=50$

\begin{tabular}{lll}
\hline Class & MHEWT & MHDWT \\
\hline 1 & 100.00 & 75.00 \\
2 & 100.00 & 100.00 \\
3 & 0.00 & 0.00 \\
Average & 66.67 & 58.33 \\
\hline
\end{tabular}

\section{CONCLUSION}

The present study investigated extraction of skeleton primitives by using skeleton subset. Though it is a tedious process of applying $2^{8}$ possibilities, it is applied and it has given a clear and accurate dominant skeleton subset. If one wants classification mainly based on skeleton subset combination, then first method of classification is more appropriate. Since all textures considered have the same dimension second method of classification is also adopted based on least skeleton points. This classification may be appropriate for shape primitive classification. By increasing lag value, number of classes will be decreased and more number of textures will be added into each class. The present method of comparison of classification rates between different nonlinear wavelets by assuming one of them as standard one is also suited for other type of texture comparison based on different classification methods. The same method can be extended to $5 \times 5,7 \times 7 \ldots \mathrm{N} \times \mathrm{N}$ masks also.

\section{ACKNOWLEDGEMENT}

The authors would like to express their gratitude to Sri K.V.V. Satya Narayana Raju, Chairman and K. Sashi Kiran Varma, Managing Director, Chaitanya group of Institutions for providing necessary infrastructure. Authors would like to thank anonymous reviewers for their valuable comments and Dr. G.V.S. Ananta Lakshmi for her invaluable suggestions which led to improvise the presentation quality of this study.

\section{REFERENCES}

1. Mallat, S., 1989. Multifrequency channel decomposition of images and wavelet models. IEEE Trans. Acoustic, Speech and Signal Process., 37:2091-2110.doi=10.1109/ICSMC.1994.400223.

2. Laine A. and J. Fan, 1993. Texture classification by wavelet packet signatures. IEEE Trans. Pattern Anal. Machine Intel., 15: 1186-1191.doi= 10.1109/IAI.2000.839620.

3. Lu, C., P. Chung and C. Chen, 1997. Unsupervised texture segmentation via wavelet transform. Pattern Recognition, 30: 729-742. http://cat.inist.fr/ ?aModele $=$ afficheN\&cpsidt $=2660327$.

4. Arivazhagan, S. and L. Ganesan, 2003. Texture classification using wavelet transforms. Pattern Recognition Letters, 24: 1513-1521. http://www.ingentaconnect.com/content/els/01678 655/2003/00000024/00000009/art00390.

5. Shutao Li and James T. Kwok, 2003. Texture classification using support vector machines. Pattern Recognition Lett., 36: 2883-2893. DOI: doi:10.1016/S0031-3203(03)00219-X .

6. Li Wang and Dong-chen He, 1990. Texture classification using texture spectrum. Pattern Recognition, 23: 905-910. doi=10.1016/00313203(90)90135-8. 
7. Eswara Reddy, B., A. Nagaraja Rao, A. Suresh and V. Vijaya Kumar, 2007. Texture classification by simple patterns on edge direction movements. IJCSNS, 7: 220-225. http://paper.ijcsns.org/07_ book/200711/20071134.pdf.

8. Krishna, V.V., V. Vijaya Kumar, U.S.N. Raju, B. Saritha, $28^{\text {th }}-30^{\text {th }}$ October 2005. Classification of textures based on distance function of linear patterns using mathematical morphology. Proceedings of ICEM, Vol.4. Conducted by JNT University, India. http://www.vedamsbooks.com/ no44382.htm.

9. Raju, U.S.N., V. Vijaya Kumar, A. Suresh and M. Radhika Mani, $27^{\text {th }}-30^{\text {th }}$ May 2008. Texture description using different wavelet transforms based on statistical parameters. In: Proceedings of the 2nd WSEAS International Symposium on Wavelets Theory and Applications in Applied Mathematics, Signal Processing and Modern Science (WAV '08), Istanbul, Turkey, pp: 174-178. http://portal.acm.org/citation.cfm?id=1404119.

10. Suresh, A., U.S.N. Raju, A. Nagaraja Rao and V. Vijaya Kumar, 2008. An innovative technique of marble texture description based on grain components. Int. J. Comput. Sci. Network Sec., 8: 122-126. http://paper.ijcsns.org/07_book/200 802/20080216.pdf.

11. Vijaya Kumar, V., B. Eswara Reddy, U.S.N. Raju and A. Suresh, 2008. Classification of textures by avoiding complex patterns. J. Comput. Sci., 4(2): 133-138. http://www.scipub.org/fulltext/jcs/jcs42 133-138.pdf.

12. Vijaya Kumar, V., B. Eswara Reddy, U.S.N. Raju and K. Chandra Sekharan, 2007. An innovative technique of texture classification and comparison based on long linear patterns. J. Comput. Sci., 3: 633-638. http://www.scipub.org/fulltext/jcs/ jcs38633-638.pdf.

13. Vijaya Kumar, V., B. Eswara Reddy and U.S.N. Raju, 2007. A measure of patterns trends on various types of preprocessed images. IJCSNS, 7: 253-257. http://paper.ijcsns.org/07_book/20 0708/20070836.pdf.

14. Vijaya Kumar, V., U.S.N. Raju, K. Chandra Sekaran and V.V. Krishna, 2008. A new method of texture classification using various wavelet transforms based on primitive patterns. ICGST Int. J. Graphics, Vision Image Process., GVIP, 8: 21-27. http://www.icgst.com/gvip/Volume8 /Issue2/ P1150819004.pdf.
15. Loncaric, S., 1998. A survey of shape analysis techniques. Pattern Recognition, 31: 983-1001. doi=10.1.1.24.138.

16. Lam, L., S.W. Lee and C.Y. Suen, 1992. Thinning methodologies a comprehensive survey. IEEE Trans. Pattern Anal. Mach. Intell., 14: 869-885. doi $=10.1109 / 34.161346$.

17. Smith, R.W., 1987. Computer processing of line images: A survey. Pattern Recognition, 20: 7-15. http://portal.acm.org/citation.cfm?id=31216.

18. Chang, H.-S., Yan, H., 1999. Analysis of Stroke Structures of Handwritten Chinese characters. IEEE Trans. Sys., Volume 29, Issue 1, pp.47 - 61 . doi $=10.1109 / 3477.740165$.

19. Saint-Marc, P., Rom, H., Medioni, G.,1993. Bspline contour representation and symmetry detection. IEEE Trans. Pattern Anal. Mach. Intell. 15, pp.1191-1197. http://doi.ieeecomputer society.org/10.1109/34.244680.

20. Zou, J.J. and H. Yan, 2001. Skeletonization of ribbon-like shapes based on regularity and singularity analyses. IEEE Trans. Syst. Man Cybern. 31: 391-395. doi= 10.1109/3477.931528.

21. Lam, L., S.W. Lee and C.Y. Suen. 1992. Thinning methodologies-a comprehensive survey. IEEE Trans. Pattern Anal. Machine Intel., 14: 869-885. doi $=10.1109 / 34.161346$.

22. Bruekers, F.A.M.L. and A.W.M. van den Enden, 1992. New networks for perfect inversion and perfect reconstruction. IEEE J. Selected Areas Commun., 130-137. doi= 10.1109/49.124464.

23. Heijmans, H.J.A.M. and J. Goutsias, 2000. Nonlinear multiresolution signal decomposition schemes-Part II: morphological wavelets. IEEE Trans. Image Process., 9: 1897-1913. http://cat.inist.fr/?aModele $=$ afficheN \& cpsidt $=1522$ 549.

24. Claypoole, R.L., Jr. Baraniuk, R.G. Nowak, R.D., May $12^{\text {th }}-15^{\text {th }} 1999$. Adaptive wavelet transforms via lifting. Proceedings of IEEE International conference at Department of Electrical and Computer Engineering, Rice University, Houston, Texas., Vol.3, pp.1513-1516. doi $=10.1109 /$ ICASSP.1998.681737.

25. Heijmans, H.J.A.M. and J. Goutsias, 1998. Morphology based perfect reconstruction filter banks. In: Proceedings of the IEEE-SP International Symposium on Time-Frequency and Time-Scale Analysis, Pittsburgh, Pennsylvania, October 6-9, pp: 353-356. doi= 10.1109/TFSA. 1998.721434. 
26. Sweldens, W., A.F. Lain and M. Unser, 1995. The lifting scheme: A new philosophy in biorthogonal wavelet constructions. Wavelet Appl. Signal Image Process. III, Proceedings of SPIE, 2569: 68-79. http://achille.research.bell-labs.com/cm/ms/who/wi m/papers/spie95.ps.gz

27. Sweldens, W., 1996. The lifting scheme: A custom-design construction of biorthogonal wavelets. Applied Comput. Harmonic Anal.,Vol. 3, 186-200. http://www.ingenta connect.com/ap/ha/1996/00000003/00000002/art00 015
28. Brodatz, P., 1966. Textures A Photographic for Artists and Designers. I ${ }^{\text {st }}$ Edition, Dover, NY.

29. Hiremath, P.S. and S. Shivashankar, 2006. Wavelet based features for texture classification. GVIP J., 6: 55-58. http://www.icgst.com/gvip/Volume6/ Issue3 /P1150636002.pdf. 